\title{
Heartbeat: the potential power of naps for cardiovascular health
}

The significance of napping for cardiovascular disease (CVD) has been a matter of controversy, with studies showing discordant results depending on the methods of evaluation, nap duration, age range of the population and outcomes measured (total vs cardiovascular mortality or events). In this issue of Heart, Häusler and colleagues ${ }^{1}$ studied the association between napping, characterised by weekly nap frequency and daily nap duration, and fatal and nonfatal CVD events in a Swiss populationbased cohort (CoLaus). Subjects $(n=3462)$ with no previous history of CVD reported their weekly nap frequency and daily nap duration, and were followed over 5.3 years for fatal and non-fatal CVD events. The authors initially observed a J-shape curved relationship between nap frequency and CVD events, with subjects napping one to two times weekly having half of the risk compared with non-napping subjects in unadjusted and adjusted models (HR $0.52,95 \%$ CI 0.28 to 0.95 ). Although there was an apparent higher CVD risk for subjects napping six to seven times weekly, this difference disappeared in models adjusted for major CVD risk factors (HR 0.89 , 95\% CI 0.58 to 1.38 ). Neither obstructive sleep apnea nor excessive daytime sleepiness modified this pattern of risk and no association was found between nap duration and CVD events. These data focus our attention on nap frequency as the key factor mediating the association between napping and cardiovascular risk, suggesting that occasional and frequent napping may have a different physiological effects and prognostic significance (figure 1).

In the companion editorial, Leng and Yaffe $^{2}$ stress that 'while it remains premature to conclude on the appropriateness of napping for maintaining optimal heart health, the findings by Häusler et al offer some reassurance that the answer is probably more than a simple 'yes' or 'no', and that we have much more to learn about napping'. 'While there remain to be more questions than answers, it is time to start

${ }^{1}$ Internal Medicine, Universidade Federal de Minas Gerais, Belo Horizonte, Brazil

${ }^{2}$ Division of Cardiology, University of Washington, Seattle, Washington, USA

Correspondence to Professor Catherine M Otto, Division of Cardiology, University of Washington, Seattle, WA 98195, USA; cmotto@uw.edu

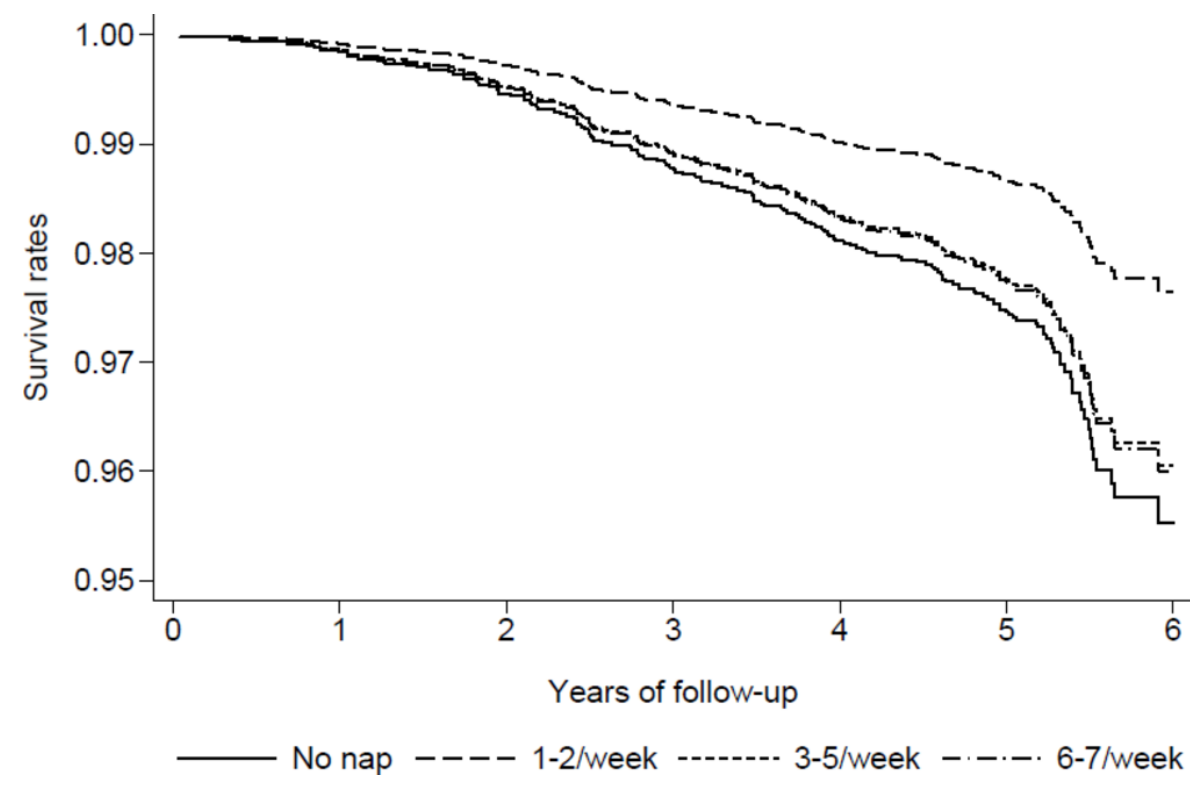

Figure 1 Survival curve, according to nap frequency.

unveiling the power of naps for a supercharged heart.'

The association of severe tricuspid regurgitation (TR) with adverse cardiovascular outcomes is firmly established. What is less clear is whether TR causes those adverse outcomes or is simply a marker of increased risk. Specifically, the clinical question is whether a reduction in TR severity would result in an improvement in cardiovascular outcomes. In a single-centre,

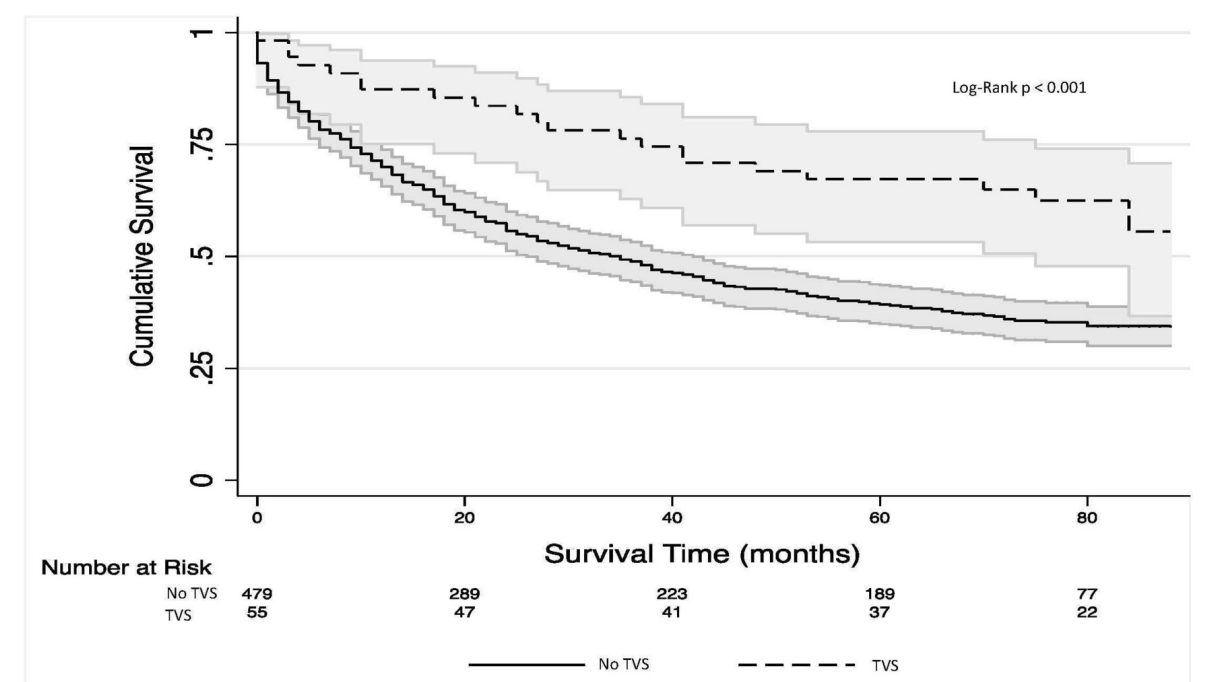

Figure 2 Kaplan-Meier curve for survival according to TVS patient group. TVS, tricuspid valve surgery.

retrospective, observational study of 534 heart failure patients with severe TR, the 55 patients (10\%) who underwent tricuspid valve surgery had a higher survival than those managed medically $\left(62 \%\right.$ vs $35 \%$; p <0.001). ${ }^{3}$ Multivariate predictors of higher mortality were age and right ventricular systolic dysfunction. In this non-randomised study, tricuspid valve surgery was associated with a lower mortality (HR: 0.44; 95\% CI 0.27 to 0.71 ) although there was no difference in 
recurrent hospital admissions for heart failure (figure 2).

In a set of two linked editorials Bernard and $\mathrm{Hung}^{4}$ first advise that 'While compelling, these results should be interpreted with caution. As pointed out by the authors, the reasons for why patients did not receive surgical intervention were not delineated. Moreover, important differences in baseline characteristics of patients who underwent surgical intervention should be noted, including their younger age, greater frequency of left-sided valvular disease and lower prevalence of heart failure with reduced ejection fraction.' Reddy and Nishimura ${ }^{5}$ review the clinical heterogeneity in patients with severe TR and the challenges in identifying which patients might respond to a mechanical intervention. They also suggest that 'atrial fibrillation-induced TR appears to represent a fundamentally different pathophysiology than other forms of secondary TR, with greater basal dilation and annular enlargement, compared with right ventricular elongation with leaflet tethering' and they propose a clinical approach based on the aetiology of TR (figure 3).

There is little data to guide indications for cardiac implantable electronic devices (CIEDs) in patients with Fabry cardiomyopathy. In 90 patients with Fabry cardiomyopathy who had undergone CIED placement, Vijapurapu and colleagues ${ }^{6}$ found that $28 \%$ had sustained ventricular tachycardia (VT) requiring antitachycardia pacing or defibrillation with an additional 26\% having non-sustained VT requiring only medical therapy. Linhart and Havranek ${ }^{7}$ remind us that Fabry cardiomyopathy differs from sarcomeric hypertrophy cardiomyopathy and 'is usually characterised by progressive diffuse left ventricular hypertrophy (LVH) with low frequency of LV outflow tract obstruction (LVOTO). Later stages are associated with inflammation, accelerated apoptosis and development of progressive myocardial replacement fibrosis affecting typically mid-wall layer of posterolateral basal LV segments (figure 4) and interstitial fibrosis, potentially also within the conduction system.' The data in the current study emphasise the high frequency of VT in these patients; the role of CIEDs deserves further evaluation.

The Education in Heart article in this issue discusses the clinical utility and approach to imaging myocardial fibrosis. ${ }^{8}$ Both a detailed table and images compare typical cardiac magnetic resonance fibrosis findings in for common types of myocardial disease. ${ }^{8}$

Our Cardiology in Focus article in this issue summarises the research of the winner and finalists for the 2019 British Cardiovascular Society Young Investigator Award: Donna Page, Naveed Akbar, Sonali

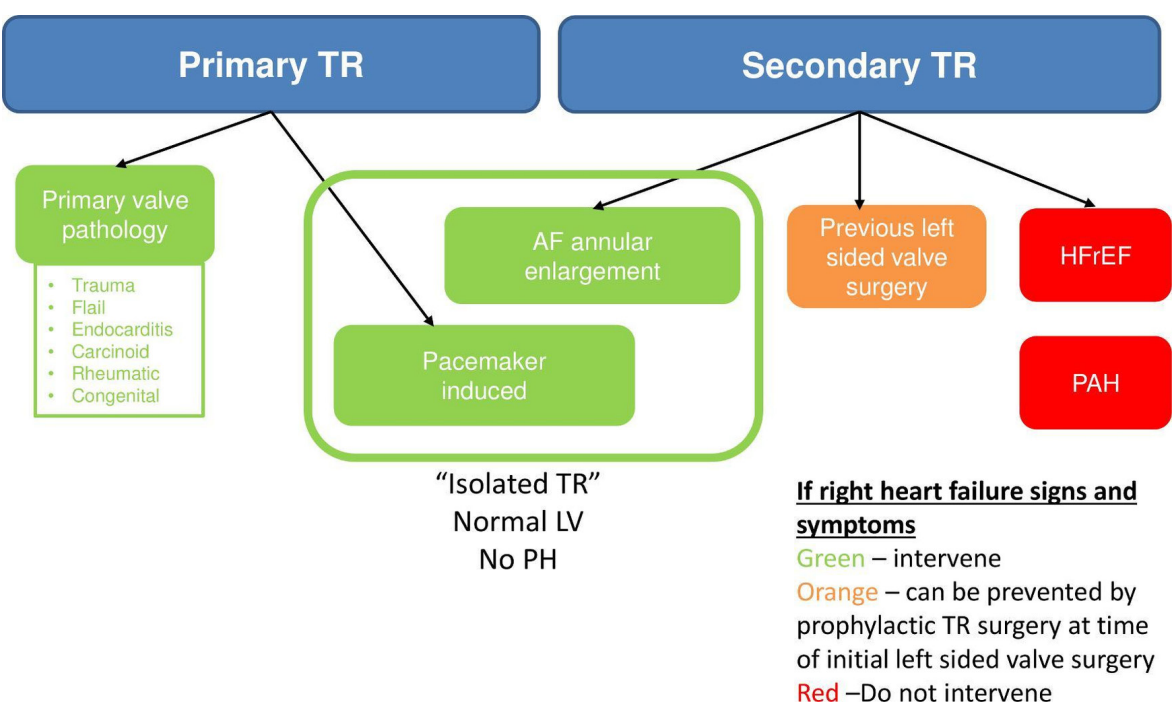

Figure 3 Clinical classification of severe TR. Severe TR can be either (1) primary from valve pathology or (2) secondary from myocardial/pulmonary vascular disease (HFrEF, PAH or prior left sided valve surgery). Patients with pacemaker-induced TR frequently have underlying myocardial disease, but this represents a form or primary TR requiring intervention. In contrast, atrial fibrillation (AF)-related TR arises secondary to annular enlargement with normal leaflets and represents a form of secondary TR, but due to its unique pathophysiology is amenable to intervention. HFrEF, heart failure with reduced ejection fraction; $\mathrm{PAH}$, pulmonary arterial hypertension; TR, tricuspid regurgitation.

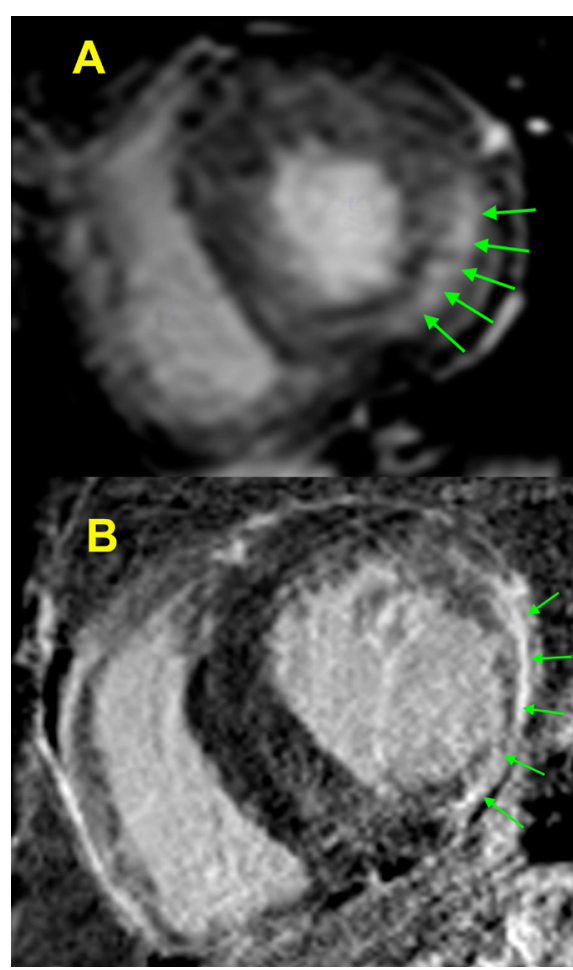

Figure 4 Cardiac MRI showing scars (green arrows) by late gadolinium enhancement in patients with Anderson-Fabry disease (AFD). Patient $A$ is a 54-year-old man classically affected with no history of palpitations or syncope. Patient B is a 52-year-old man with later-onset variant scanned after being resuscitated from sudden cardiac death. of note is the greater extent of fibrosis and excessive thinning of affected myocardial segments. Images courtesy to general university hospital in Prague.

Munshaw and Betty Raman. ${ }^{9}$ We hope to see many future excellent research publications from these outstanding young investigators!

Funding The authors have not declared a specific grant for this research from any funding agency in the public, commercial or not-for-profit sectors.

Competing interests None declared.

Patient consent for publication Not required.

Provenance and peer review Commissioned; internally peer reviewed.

(C) Author(s) (or their employer(s)) 2019. No commercial re-use. See rights and permissions. Published by BMJ.

\section{(D) Check for updates}

To cite Ribeiro AL, Otto CM. Heart 2019;105:1765-1767.

Heart 2019;105:1765-1767. doi:10.1136/heartjnl-2019-316158

\section{ORCID iDs}

Antonio Luiz Ribeiro http://orcid.org/0000-0002-27400042 
Catherine M Otto http://orcid.org/0000-0002-05279392

\section{REFERENCES}

1 Häusler N, Haba-Rubio J, Heinzer R, et al. Association of napping with incident cardiovascular events in a prospective cohort study. Heart 2019;105:1793-8

2 Leng Y, Yaffe K. To nap or not to nap: more questions than answers. Heart 2019;105:1768-9.
3 Kadri AN, Menon V, Sammour YM, et al. Outcomes of patients with severe tricuspid regurgitation and congestive heart failure. Heart 2019;105:1813-7.

4 Bernard S, Hung J. Managing tricuspid valve regurgitation: a long and winding road. Heart 2019:105:1773-4

5 Reddy YNV, Nishimura R. Intervening for tricuspid regurgitation: uncertainties in a heterogeneous syndrome. Heart 2019;105:1770-2.

6 Vijapurapu R, Geberhiwot T, Jovanovic A, et al. Study of indications for cardiac device implantation and utilisation in Fabry cardiomyopathy. Heart 2019;105:1825-31.

7 Linhart A, Havranek S. Conundrum of implantable electric devices in Anderson-Fabry cardiomyopathy. Heart 2019;105:1775-6.

8 Bing R, Dweck MR. Myocardial fibrosis: why image, how to image andclinical implications. Heart 2019:105:1832-40.

9 Joanne D, Akbar N, Munshaw S. British cardiovascular Society young investigator Award 2019. Heart 2019;105:1841-2. 